\title{
ethic@ \\ MENTES NO BOLSO: \\ CONSIDERAÇÕES NEUROÉTICAS SOBRE A \\ INCORPORAÇÃO DE APLICATIVOS DE SMARTPHONES NA CONFIGURAÇÃO DO SELF
}

\author{
MINDS IN THE POCKET: \\ NEUROETHICAL CONSIDERATIONS ABOUT THE \\ INCORPORATION OF SMARTPHONE APPLICATIONS IN THE \\ CONFIGURATION OF THE SELF
}

DIOGO GONÇALVES VIANNA MOCHCOVITCH ${ }^{1}$

(UFRJ/Brasil)

MARIA CLARA DIAS ${ }^{2}$

(UFRJ/Brasil)

\begin{abstract}
RESUMO
A neurociência e os estudos da Interação Humano-Computador crescem exponencialmente. Defende-se que a realização dos processos cognitivos não depende apenas do cérebro, mas também da interação entre o agente cognitivo e diversos artefatos e, por isso, seres humanos podem ser considerados ciborgues de nascença. A relação simbiótica que temos com nossos gadgets, em especial, o smartphone, lança nova luz sobre problemas neuroéticos surgidos com o uso das tecnologias de informação e comunicação, TIC. Nosso objetivo neste artigo é (1) contextualizar o uso de tecnologias digitais como extensões da mente, em especial smarthphones, e (2) discutir as questões neuroéticas provenientes desse uso. Argumentamos que as sugestões de aplicativos, através de seus algoritmos, bem como o uso de nossas informações, podem ser consideradas alterações em nossas mentes e, consequentemente, no nosso self. Por isso, procuramos, (3) oferecer algumas diretrizes para que o debate possa assumir um caráter mais preventivo para os problemas emergentes.
\end{abstract}

Palavras-chave: Neuroética; Tecnologia; Smartphone, Algoritmos; Self.

\begin{abstract}
Neuroscience and Human-Computer Interaction studies are growing exponentially. It is argued that the realization of cognitive processes does not depend only on the brain, but also on the interaction between the cognitive agent and various artifacts and, therefore, human beings can be considered natural-born cyborgs. The symbiotic relationship we have with our gadgets, especially the smartphone, sheds new light on neuroethical problems that have arisen with the use of information and communication technologies, ICT. Our aim in this article is (1) to contextualize the use of digital technologies as extensions of the mind, in particular smarthphones, and (2) to discuss the neuroethical issues arising from this use. We argue that the suggestions of applications, through their algorithms, as well as the
\end{abstract}


use of our information, can be considered changes in our minds and, consequently, in our self. For this reason, we seek, (3) to offer some guidelines so that the debate can take on a more preventive character for emerging problems.

Keywords: Neuroethics; Technology; Smartphone, Algorithms; Self.

\section{Introdução}

A neurociência e os estudos da Interação Humano-Computador crescem exponencialmente. Na literatura da filosofia da mente contemporânea, diversos autores defendem que a realização dos processos cognitivos não depende apenas do cérebro, mas também da interação entre o agente cognitivo e diversos artefatos (CLARK; CHALMERS, 1998; DENNETT, 1996; WILSON, 2004) e, por isso, seres humanos podem ser considerados ciborgues de nascença (CLARK, 2003).

A relação simbiótica que temos com nossos gadgets, em especial, o smartphone, aponta nova luz sobre problemas neuroéticos surgidos com o uso das tecnologias de informação e comunicação, TIC. Nosso objetivo neste artigo é (1) contextualizar o uso de tecnologias digitais como extensões da mente, em especial smartphones, e (2) discutir as questões neuroéticas provenientes desse uso. Defendemos que as sugestões de aplicativos, através de seus algoritmos, bem como o uso de nossas informações, podem ser consideradas alterações em nossas mentes e, por conseguinte, do nosso self. Questões sobre o controle da informação, algoritmos modelados segundo a resposta do usuário, sistemas de recomendação (recommendation systems) via inteligência artificial, necessitam de uma abordagem analítica. Por isso, procuramos (3) oferecer algumas diretrizes para que o debate possa assumir um caráter mais preventivo para os problemas emergentes.

Inicialmente, é necessário contextualizar o cenário de onde partimos para, em seguida, analisar como nossos smartphones podem ser considerados parte da nossas mentes. Os debates atuais nas áreas da ciência cognitiva e filosofia da mente são herança de duas tradições fenomenológicas: a fenomenologia tradicional de Husserl (2000) e MerleauPonty (2006) e a abordagem ecológica de James Gibson (1986). No escopo de suas trajetórias teóricas, entre suas aproximações e distanciamentos, ambas as teorias defendem que o processamento da informação da mente depende, necessariamente, do ambiente, formando uma ecologia cognitiva (LÉVY, 1993). O resultado disso é o crescente distanciamento do pensamento cartesiano que defende a dicotomia entre mente (sujeito) e mundo (objeto).

Neste artigo, adotaremos a tese da mente estendida (CLARK; CHALMERS, 1998; CLARK, 2010) como ponto de partida da nossa investigação. Esta posição defende que a mente está para além das barreiras corporais e o processo cognitivo pode ser realizado também 
através de veículos epistêmicos externos. Em última instância, a tese da mente estendida afirma que, em alguns casos, é possível considerar tais objetos, sejam digitais ou analógicos, como um sistema acoplado (CLARK, 2001), que se torna parte constitutiva de um sistema mais amplo, para realização do processo cognitivo ${ }^{3}$.

Sem dúvida, nem todo artefato usado por um agente cognitivo será considerado um sistema acoplado. Clark (CLARK, 2010) estabeleceu uma série de critérios de segurança (glue and trust) para determinar um acoplamento bem-sucedido:

1. That the resource be realibly available and tipically invoked.

2. That any information thus retrieved be more or less automatically endorsed. It should not usually be subject to critical scrutiny. [...] It should be deemed about as trustworthy as something retrieved clearly from biological memory.

3. That information contained in the resouce should be easily accessible as and when required (CLARK, 2010, 46, grifo nosso).

Com o quadro acima apresentado, o agente cognitivo poderá considerar algo como um sistema acoplado caso o artefato esteja (1) disponível de forma confiável, (2) tenha endosso automático e (3) seja facilmente acessível. Neste sentido, um sistema acoplado pode ser tanto digital quanto analógico. Um bloco de anotações para um escritor participa ativamente da economia cognitiva e de seus processos mentais, bem como um artefato digital. Em sentido amplo, podemos considerar que esse critério determina que as ferramentas mentais devam permitir o uso transparente (CLARK, 2003), de forma que o agente cognitivo visualize a resolução do problema diretamente e não a manipulação do artefato ou o questionamento acerca da sua confiabilidade.

A tese da mente estendida está em pleno debate e constitui um dos temas mais discutidos na filosofia da mente contemporânea, neuroética, ciência cognitiva em interface com a web science e comunicação. Há autores que discordam dessa teoria (ADAMS; AIZAWA, 2001; RUPERT, 2009), alegando que esse conceito provoca um tipo de inchaço cognitivo (cognitive bloat). Isto é, aceitar uma tese que assume veículos externos epistêmicos como parte do processo cognitivo e, por conseguinte, da mente, pode fornecer estatutos ontológicos equivocados a diversos artefatos.

Críticos do conceito de mente estendida defendem que as funções cognitivas estão sob responsabilidade da nossa estrutura biológica, mais particularmente, do cérebro. Por outro lado, há cada vez mais evidências de que as novas tecnologias têm assumido a gestão desses processos. Os smartphones, em especial, já são considerados wearables (KREITMAIR; $\mathrm{CHO}, 2017)$, isto é, dispositivos vestíveis, sempre disponíveis devido ao seu uso quase ininterrupto e sua presença em nossas rotinas cognitivas. $O$ 
advento do uso desse tipo de tecnologia ${ }^{4}$, sua influência em decisões, descarregamento de informações e rememoração através de nossos dispositivos móveis, modelam grande parcela de nossas atividades na atualidade. Desta maneira, os usuários de smartphones têm fluência na operacionalização da tecnologia, isto é, os sujeitos que utilizam seus artefatos tecnológicos visualizam diretamente a tarefa a ser resolvida, tal como pressupõe o uso transparente (CLARK, 2003), acima mencionado como um critério para que um dispositivo possa ser caracterizado como uma extensão da mente.

Não cabe no escopo deste texto situar esse debate para defender todas as nuances envolvidas na argumentação da teoria da mente estendida. Para o propósito deste texto, cabe ressaltar que a tecnologia atual, wearables, smartphones e a Internet of Things (Internet das coisas), contribuem no cálculo mental, aumentando a velocidade de processamento e são parte crucial da nossa vida cognitiva5. Aplicando o critério de acoplamento de Clark para a mente estendida, o aparelho smartphone satisfaz essas condições, já que está disponível de forma confiável e também é tipicamente invocado; é endossado automaticamente para operar diversas rotinas cognitivas e as informações contidas nele são acessíveis.

O cerne do argumento indica que esses artefatos não só nos auxiliam como muletas cognitivas, mas também carregam nossas intenções, crenças e preferências. Esses estados mentais são comumente reportados como parte essencial de nossa memória biológica. À medida que nossos artefatos tecnológicos se personalizam - adequando-se diariamente às nossas rotinas, experiências e deliberações - mais passam a ser percebidos como parte constitutiva das nossas mentes.

Por conta das características apresentadas, a relação entre agentes cognitivos e artefatos tecnológicos é classificada como tecnologias da mente estendida (TMEs) (REINER; NAGEL, 2017). Uma TME pode, por sua vez, se tornar parte da nossa identidade pessoal, o self. Defendemos neste artigo que o self é um tipo de unidade narrativa de constituição autobiográfica. Para um sujeito se reportar como uma unidade narrativa não é necessário que o indivíduo conscientemente reflita sobre a história de sua vida, tal como a narrativa de um romance, mas sim que ele se situe e possa se identificar, utilizando as principais características constitutivas (SCHECHTMAN, 1996) de sua personalidade. ${ }^{6}$

Assim, devido à onipresença e à influência em nossos processos cognitivos, as TMEs assumem um grau de relevância e responsabilidade de grande parcela das atividades realizadas pelo self. Tomemos como exemplo o caso de um usuário padrão de smartphone que insere os números de telefones e e-mails de seus contatos em sua agenda telefônica, bem como cria listas de suas tarefas diárias e escreve ideias para seu livro através do seu aparelho móvel. O gatilho para a rememoração e o acesso aos dados fica depositado no meio digital, em uma nuvem, à espera de sua rememoração. Essa situação, para Clark e Chalmers, é muito mais que uma analogia. Tal como crenças disposicionais, esse tipo de informação também está à disposição para seu acesso. Dada as rotinas cognitivas do processo 
de rememoração, um sujeito pode buscar informações em sua plataforma digital, sua memória estocada, deliberadamente guardada para posterior acesso ${ }^{7}$.

$\mathrm{Na}$ esteira deste pensamento, Carter e Palermos indicam o amálgama entre biologia e tecnologia:

As our daily reliance on certain artifacts becomes increasingly prevalent, it may become impossible to count all extra-organismic possessions as distinct to our personhood, and thereby merely as our property" (CARTER; PALERMOS, 2016, 546).

Esse tipo de comparação não é muito distinto da situação de um escritor que sempre carregue consigo um bloco de papel e uma caneta no bolso de sua camisa para anotar ideias.

Reiner e Nagel apresentam uma nova perspectiva:

(...) there is a relatively seamless interaction between brain and algorithm such that a person perceives of the algorithm as being a bona fide extension of a person's mind. This raises the bar for inclusion into the category of algorithms that might be considered TEMs. It is also the case that algorithmic functions that do not qualify as TEMs today may do so at some future point in time and vice versa (REINER; NAGEL, 2017, 110).

A afirmação acima ultrapassa a hipótese inicial de Clark e Chalmers, pois não somente os artefatos são parte constitutiva da mente, mas os algoritmos dos aplicativos que utilizamos em nossos smartphones podem, em pouco tempo, receber um status ontológico distinto. Decerto, o uso dos aplicativos também requererá os mesmos critérios de segurança apresentados anteriormente. Uma vez que um usuário utilize um aplicativo de maneira bastante modesta do ponto de vista operacional e hesite diversas vezes no seu uso, não confie e não tenha disponibilidade, esse algoritmo não poderá ser considerado como uma TME. Contudo, conforme esse usuário se familiarize com o aplicativo, inicie uma rotina de uso, e forneça informações ao aplicativo, cada vez mais o usuário irá manipular melhor o aplicativo, receberá feedbacks que se tornam mais pertinentes às necessidades que o sujeito demanda ao algoritmo.

Diante do exposto, chegamos, portanto, ao objetivo central deste artigo. Após a revisão bibliográfica que estabelece que os nossos smartphones são, em alguns casos, parte (primordial algumas vezes) de nossas mentes, é imprescindível analisar as implicações que as modificações, sugestões e/ou intromissões em nossos aparatos tecnológicos podem causar às nossas mentes e, também, ao self.

Trataremos de algumas questões para esse debate que são centrais e que estão completamente interligadas, representando a divisão de seções 
apenas uma arbitrariedade sistemática para fins de explanação: autenticidade e autonomia (poderíamos considerar que as sugestões fornecidas pelos algoritmos via inteligência artificial em nossos gadgets e wearables são intromissões em nossa mente ou apenas uma forma mais explícita de apresentar nossos desejos?); self quantificado e responsabilidade (a forma como são realizadas as categorizações e operacionalizações via aplicativos podem ser seguras para a construção da identidade pessoal? A quem podemos responsabilizar, caso indicações de aplicativos não ocorram de forma satisfatória?); por último, analisaremos as questões que já são consideradas clássicas: privacidade, transparência e uso de dados sem consentimento.

\section{Autenticidade e Autonomia}

Para corroborar com a investigação em curso, destacaremos algumas hipóteses extraídas da literatura recente, mas, antes de analisá-las, é necessário tecermos alguns comentários sobre o conceito de mente. Entre as principais características dos processos mentais estão a privacidade dos conteúdos mentais e o acesso privilegiado. Isto é, um sujeito que tenha a crença "A cidade de São Paulo é cosmopolita", já dispõe de um saber acerca dela antes de proferi-la, pois tem armazenado em seu "hardware" biológico essa crença e possui acesso privilegiado a essa estrutura ${ }^{8}$. De forma análoga, o principal atributo para um self narrativo são as características idiossincráticas que norteiam o sujeito através da autenticidade e autonomia de seus desejos. Isto é, as ações realizadas por um sujeito são fruto de estados mentais que se alinham com suas vontades ${ }^{9}$.

Caso levemos a sério a discussão supracitada, devemos considerar que as sugestões - sejam devidas ou indevidas - de sistemas de recomendação estão diretamente envolvidas na forma que um sujeito conduz sua vida. Reiner e Nagel (2017) descrevem uma situação para entendermos o uso do GPS como uma TME. Imaginemos que John sempre se locomoveu pela cidade de Nova Iorque a pé, utilizando os sistemas de transportes (ônibus e metrô) como principal maneira de ir aos locais mais distantes da cidade. Por contingências da vida, ele precisa aumentar sua renda e pediu o carro de seu irmão emprestado para trabalhar durante as noites dirigindo através do Uber, um aplicativo de prestação de serviço de motoristas não profissionais.

Nas primeiras vezes que John utilizou o carro em conjunto com o GPS ficou perplexo como era possível navegar com facilidade por locais onde jamais teria a possibilidade de estar somente com sua memória ou seu antigo mapa mental das linhas de metrô ou rotas de ônibus da cidade. Ainda que estivesse bastante empolgado com o uso do GPS, John ainda tinha certa dificuldade em percorrer as rotas indicadas, já que suas respostas baseadas nas indicações do aplicativo ainda eram lentas e vagamente incrédulas.

Após duas semanas de utilização ininterruptas, o aplicativo já era muito mais fluído para John, além de muito mais confiável. Suas respostas eram automáticas e o motorista não precisava mais visualizar todas as 
indicações na tela de seu smartphone, pois existia entre o agente cognitivo, aparelho e aplicativo um acoplamento tão fluido que John visualiza a realização da tarefa diretamente. Em outras palavras, após o período de conformação de John, seu aparelho e o aplicativo, o condutor se concentra em chegar ao destino de seu passageiro e está integrado ao aplicativo de tal forma que não precisa pensar em cada etapa das instruções do GPS

Os autores levantam uma hipótese sobre a rotina cognitiva que envolve o uso do GPS, um sistema de recomendação e o motorista. John, ao iniciar seu trabalho como motorista de Uber, recebe em seu GPS a sugestão de que há uma confeitaria próxima de sua localização atual. Alguns minutos passados e passageiros conduzidos aos seus destinos, John para o carro em uma confeitaria para comer um donut.

Em uma situação similar (REINER; NAGEL, 2017), imaginemos que além do seu GPS em seu smartphone, John incorpora à sua ecologia cognitiva (LÉVY, 1993) um smartwatch (relógio inteligente) integrado aos diversos aplicativos instalados em seu smartphone. Seu relógio aferiu o tempo de corrida, monitorou seus batimentos cardíacos e rastreou o percurso de sua corrida, integrando esses dados ao seu aplicativo de nutrição. A sugestão advinda do sistema de recomendação neste caso será de um pedaço de torta de uma confeitaria orgânica, uma vez que o sistema global possui uma leitura mais completa das rotinas de John, seus gostos e preferências. O aplicativo tem os registros de sua alimentação e pode lembrá-lo que seu consumo calórico foi baixo e na manhã do mesmo dia correu 10 quilômetros.

Reiner e Nagel (2017) indicam que o primeiro caso parece não se adequar a uma legítima sugestão, já que não existe uma leitura das idiossincrasias, ou de porções substanciais do self de John. Já o segundo caso parece estar integrado às preferências de John, sendo, portanto, menos invasivo ou desonesto. Carter e Palermos apontam para esta mesma direção:

(...) the emergence of personal computing, and the advent of wearable technologies and the cloud - all of which regularly store personal data automatically, in order to then structure our daily schedules, offer suggestions and monitor our health on a moment-to-moment basis - have begun to blur the lines between our biological and digital existence (CARTER; PALERMOS, 2016, 546).

Os resultados advindos do quadro elucidado fomentam a discussão sobre as noções de autenticidade e autonomia. É preciso determinar quais as consequências de um sujeito que conduz sua vida baseado em sugestões de um aplicativo. Muitas vezes sequer nos atentamos às diversas escolhas eleitas através de desejos de segunda ordem que são introjetados pouco a pouco através de nossas TMEs. Em última instância, nossos aplicativos, em quase sua totalidade, estão sob controle de grandes conglomerados tecnológicos que tem por objetivo a difusão das vendas e o estabelecimento 
de um nicho de mercado que possa progredir e alcançar o maior número de consumidores.

Reiner e Nagel afirmam que é impossível olhar para essa questão imparcialmente, pois os algoritmos estão "servindo a dois mestres" (REINER; NAGEL, 2017, 115). Neste sentido, é preciso determinar em que medida é benéfico esse tipo de abordagem realizada pelos algoritmos dos aplicativos. Segundo a abordagem apresentada, a massificação implementada, tal como o conceito de indústria cultural aos moldes adornianos, se torna mais sofisticada, pois atinge não só um potencial mercado, mas também a criação não autorizada de modificações na mente dos sujeitos.

Autenticidade e autonomia da mente - conceitos basais para a neuroética - são duas faces de uma mesma moeda que precisam estar em acordo. A autonomia sobre nossos desejos e a liberdade de realizar ações é um dos pilares para uma concepção de self, para a criação da identidade pessoal. Muitas vezes as ações passam a ser deliberadas apenas após a "aprovação" das TMEs. Os wearables de self-tracking (autorrastreamento) informam aos aplicativos de saúde, por exemplo, sobre os principais dados da saúde, de tal forma que o sujeito passa a não respeitar suas intuições, mas sim a agir apenas através dos dados (ERDENIZ et al, 2019).

Assim, as questões neuroéticas sobre a autenticidade e a autonomia se tornam prementes para a investigação, já que, conforme exposto, nossos smartphones são tecnologias da mente estendida, portanto são partes muito importantes das nossas mentes e que podem sofrer inúmeras interferências que culminam em intervenções na autenticidade e na autonomia do self. Chegamos assim à questão do self quantificado e da responsabilidade individual.

\section{Self quantificado e responsabilidade}

Analisaremos dois tipos de aplicativos que, apesar de distintos em seus propósitos, são ferramentas que tornam o self quantificado (quantified self). Entendemos como self quantificável o conjunto das ações de um indivíduo que pode vir a ser aferido quantitativamente, a partir de dados obtidos por aplicativos. Desta forma, suas ações se tornam parametrizadas e previsíveis (ERDENIZ et al, 2019), servindo para análise comparativa, seja para próprio indivíduo, seja para outros usuários ou, ainda, para a empresa criadora do aplicativo.

O primeiro tipo de aplicativo é aquele que auxilia em determinadas tarefas de monitoramento do sujeito que realiza sua rotina de exercícios e deseja obter mais controle sobre seus dados biológicos e, consequentemente, sobre o estado de sua saúde. Já o segundo tipo de aplicativo se baseia no auxílio do usuário ao autoconhecimento através de conversas que aproximam o usuário do cenário de uma sessão de terapia e/ou análise virtual com a figura de um psicólogo que, em realidade, é uma inteligência artificial.

Nossa argumentação é que mesmo que no primeiro aplicativo seja mais evidente a transformação do self em um self quantificável - já que o 
próprio sujeito consegue visualizar seus dados para fins de tomada de decisão e/ou comparação - o segundo aplicativo também parametriza identidades.

Aplicativos de saúde monitoram a performance de usuários através de wearables como smartwatches que mapeiam batimentos cardíacos, quilômetros percorridos em corridas ou caminhadas, horas de sono, quantidade de passos dados durante o dia, nível de oxigenação, perda calórica etc. Em geral, esse tipo de análise possui dois tipos de compartilhamentos pelo usuário: (1) via suas outras redes sociais e (2) via comunidade do próprio aplicativo, no qual o usuário pode trocar informações, comparar com outros usuários, além de participar de um ranqueamento entre os usuários com maior pontuação e mais atividade dentro da plataforma.

Como mencionado anteriormente, o self quantificado torna o sujeito menos propenso a realizar ações consideradas "instintivas". Colocamos as aspas para destacar que seu processo decisório que antes tinha como base sua introspecção, possui, agora, um filtro que é capaz de apresentar além de recomendações, restrições às ações subsequentes que serão ou não realizadas. Isso, em termos de vontade para ações autênticas e autônomas, cria implicações distintas do modo de se lidar com a construção das volições. Entretanto, para além dessas duas questões, há no horizonte de problemas neuroéticos a própria criação do self, bem como do outro. Ou seja, tanto para construção do eu como a para a alteridade, existem dinâmicas afetadas que corroboram para modificações que irão influenciar as interações que formam a contínua construção do self.

Em sua base narrativa, o self não possui a demanda de um lócus especial, um sítio definido e inerente apenas ao sujeito. Em uma narrativa, o sujeito participa de uma trama que pretende promover encontros, realizar ações e desenvolver sua história. Quando temos no horizonte os aparatos tecnológicos e algoritmos como as TMEs, é possível afirmar que, em grande parte, o que é essencial para a narrativa é informação ${ }^{10}$. Informação essa que está espalhada pelas nuvens dos diversos serviços, dos aplicativos nos quais despejamos nossos dados e co-criamos, juntos aos mesmos, nossas histórias.

Decerto, tecnologias analógicas já repercutiam na criação de um self quantificado. A diferença que existe com a inserção de tecnologias, em especial o smartphone, é apontada por Gabriels e Coeckelbergh (2019) através de três características: (1) constância na rede. Por mais que estejamos sem acessar o app, o wearable constantemente está monitorando o sujeito e transmitindo seus dados, mesmo quando o usuário não esteja deliberadamente autorizando e/ou consciente deste tipo de monitoramento; (2) respostas imediatas e feedbacks de seguidores, ou de uma comunidade, transformam a noção de espaço e tempo e (3) por conta do feedback quase instantâneo, é criada uma nova forma de disciplina que deve atender ao novo tipo de espacialidade e temporalidade mencionados anteriormente. 
A troca de informação entre performances não só altera a capacidade de autocompreensão do sujeito, como instaura, através dos comparativos da comunidade, plataforma, ou conexão com outros participantes de aplicativos de saúde, a maneira como ele irá lidar com a sua própria autobiografia. Isto é, esses novos parâmetros podem causar um estranhamento de si (um estranhamento do eu) que não estão mais balizados em sua própria performance, mas em uma relação comparativa, baseada apenas em números.

Ademais, essa questão da quantificação do eu e do outro merece uma segunda análise, desta vez voltada para a acessibilidade e velocidade do fluxo de informações. A velocidade das publicações de performances, o constante acesso aos resultados de outras pessoas, assim como a consequente comparação consigo, traz um nível de proximidade nunca antes experimentado. O outro passa a ser visualizado dentro da rotina de construção da narrativa do eu, isto é, ao conceber seu projeto de vida, seus anseios e desejos para traçar sua narrativa identitária, o outro assume uma importância determinante.

Em outras palavras, o outro passa a ser um dos parâmetros mais importantes da construção do eu, de tal forma que seu acesso é tão estável e corriqueiro, que torna um desafio para a trama narrativa de um sujeito não se comparar e basear seu self nas avaliações comparativas e quantificáveis. Esses tipos de relações promovem uma forma de gameficação das relações sociais "em relação aos outros que surgem mais e mais como manifestações e competição para obter melhores pontuações" (GABRIELS; COECKELBERGH, 2019, 07).

Em relação aos aplicativos de análise/terapia, aparentemente, esses poderiam ser considerados com menor teor de quantificação, entretanto, a metrificação também está posta. Vejamos como funciona a maioria desses aplicativos. Inicialmente, imaginemos um sujeito $X$ com quadro leve de depressão. Após alguns minutos navegando por sua loja de aplicativos para smartphones, se depara com um aplicativo de cunho terapêutico que pretende realizar conversas especializadas com um psicólogo ou terapeuta virtual que auxiliará no processo de melhoria da depressão de $X$.

Após a instalação do aplicativo e sua inicialização, o aplicativo precisa de algumas informações básicas e inicia uma dinâmica de conversas, trocas de textos e, também, áudios. Esse aplicativo não terá, à disposição do sujeito $X$, um profissional que auxiliará sua consulta e tratamento psicológico. Em geral, a fórmula está baseada em aprendizado de máquina (machine learning) (COECKELBERGH, 2019) que é responsável por coleta, sistematização, processamento (inclusive com outras tecnologias digitais) de informações e dados dos usuários.

Decerto que esses aplicativos possuem equipes de psicólogos que realizam consultorias para estabelecer os caminhos que devem ser estruturados para as análises e perguntas que serão realizadas no sistema, entretanto, não é o caso de ter a presença de um profissional específico para o sujeito $X$. Sua interlocução está baseada em conhecimento acumulado disposto via chatbot, um mecanismo de conversa com a IA que possui aprendizado e treinamento via programação. 
O auxílio de psicólogos em alguns aplicativos torna a experiência do aprendizado de máquina mais rápido e, também, aumenta o nível de acurácia e assertividade das análises ${ }^{11}$. Um dos temas que podem ser levados em consideração é: ao tratar com um psicólogo teríamos um atendimento particularizado, onde o profissional tem, sob seu comando, técnicas que são aprendidas na universidade para tratar uma pessoa. $\mathrm{Na}$ outra esteira de análise temos o aplicativo, através do aprendizado de máquina. Poderia ele ser tão específico no seu "tratamento" uma vez que não tem a capacidade de particularizar como nós, seres humanos?

Uma resposta prévia a essa questão por um defensor do aplicativo seria que o aprendizado sobre o sujeito em análise seria cada vez mais particularizado conforme ele entregasse mais e mais informações, da mesma forma que um psicólogo e/ou terapeuta poderia buscar em suas sessões. Outro argumento possível para defender esse tipo de abordagem é que, talvez, alguns traços que poderiam não ser notados por um psicólogo humanos poderiam ser apreendidos, decodificados e indicar novas hipóteses e/ou possibilidades aos atendimentos pelo psicólogo do aplicativo.

Por outro lado, seria possível apontar diversos pontos a favor do processo tradicional de atendimento psicológico. Sessões com psicólogos humanos possuem alguns pontos positivos como uma relação de empatia, com o compartilhamento de certas vivências sociais, o acordo de confidencialidade entre paciente e psicólogo, além de um espaço de confiança mútua.

Nosso intento não é realizarmos comparações maniqueístas e binárias entre humanos versus máquina, mas apenas levantar algumas questões que implicarão em encaminhamentos específicos para o estabelecimento de diretrizes éticas concernentes à identidade pessoal.

No cenário acima descrito, em comparação com o aplicativo de monitoramento de saúde, parece que ainda não existem métricas suficientes para afirmarmos que esse tipo de aplicativo para auxílio psicológico seja também um possível estimulador do self quantificado. Contudo, pela própria natureza categorial do aplicativo, e pelo recolhimento de dados ou de acessos que pode ser concedido ao instalá-lo no aparelho celular do usuário é possível apontarmos outros argumentos.

As questões de Big Data e Data Analytics podem nos apontar como é possível tratar essa temática. Com a crescente absorção de informações de usuários, os bancos de dados dos aplicativos das empresas de tecnologia estão cada vez mais robustos e capazes de agregar mais informações e, portanto, detalhes dos usuários. Assim, suponhamos que o sujeito $\mathrm{X}$ esteja utilizando o aplicativo de auxílio psicológico. Poderia esse aplicativo, pela sua própria característica, categorizar e enquadrar o sujeito $\mathrm{X}$ em algum modelo de usuário e, a partir de outras métricas, iniciar sugestões que recaem, novamente, na questão da autenticidade e na autonomia de um indivíduo.

Nosso argumento nesta etapa é de que, por se tratar de uma abordagem de cunho generalista pelo aplicativo, sua própria forma de 
recomendação seria baseada nas mesmas duas situações apresentadas anteriormente no caso do aplicativo Uber. No primeiro momento, o aplicativo poderia ser mais hesitante, mas, após receber mais respostas e os feedbacks do sujeito $\mathrm{X}$, o aprendizado estaria mais acurado e pronto a ser mais personalizável para o usuário.

A questão neste caso de aplicativo é o caráter particularista, inesperado e, principalmente, criativo, de seres humanos que pode ser um desafio às consultas de cunho terapêutico/psicológico. A dificuldade de quantificação reside, primordialmente, nesse quesito. As respostas dos aplicativos, ainda que sejam bastante elaboradas, não superam nossas particularidades e criatividade.

Nos casos que envolvem autoconhecimento e a continuação da criação da narrativa autobiográfica de um sujeito é necessário que existam regras para entendermos até que ponto os algoritmos poderão ter acesso e ajudarão na formação de porções cada vez maiores da trajetória da identidade pessoal, ou, inversamente, atrapalharão e ainda serão considerados invasores de privacidade de conteúdos mentais.

Neste sentido, o que em princípio poderia soar como trocas e leituras circunstanciadas do self pode, também, realizar influência determinante na vida de alguém por conta de uma leitura parametrizada e, portanto, quantificável de um sujeito. Principalmente quando se trata de lidar com a criatividade de seres humanos. Poderíamos ter em algum tipo desses aplicativos, por conta de uma dificuldade de interpretação do sistema de crenças de alguma pessoa, recomendações que irão indicar caminhos que poderiam limitar a narrativa do sujeito.

Quando o aprendizado de máquina é associado ao aprendizado profundo (deep learning), no qual o sistema não segue mais as indicações da programação previamente estipulada, mas realiza um aprendizado por conta própria, o resultado pode ser ainda mais desafiador. Instaura-se uma espécie de caixa preta onde o acesso para possíveis ajustes de programação que podem resultar em um processo de enviesamento determinado por parte do sistema operante. Tal ponto, associado a um aplicativo que está diretamente lidando com o self, torna o cenário ainda mais complexo.

Examinemos, então, até as últimas consequências a questão da responsabilidade. A quem será atribuída a responsabilidade caso o sujeito $X$ receba sugestões que possam iniciar um processo de trauma psicológico? No caso desse aplicativo genérico, encontrado em larga escala no mercado de tratamento psicológico, a quem atribuiremos a responsabilidade: aos programadores, à equipe de psicólogos que podem realizar consultorias ao aplicativo ou à empresa responsável pela criação do aplicativo? Esse problema da responsabilidade moral apresenta a dificuldade conhecida na literatura como o dilema das "muitas mãos" (COECKELBERGH, 2019):

Since technologies cannot be responsible moral agents and are hence a-responsible, the only way to ensure responsible action is to make humans responsible. However, in technological action it is notoriously difficult to ascribe moral responsibility due to the so-called problem of "many hands": 
many people are involved in the often long causal histories that lead to a particular outcome. If there is a problem with the end result, say a recommendation, it is difficult to figure out who was responsible. And since AI is often part of a larger technological system and data histories, it is difficult to figure out if "the AI" caused the problem or some other part of the system. There are not only many hands but also many things (COECKELBERGH, 2019, 31).

Não pretendemos com a explanação acima defender um argumento tecnofóbico, na promoção de políticas que impeçam a criação desses aplicativos. Entretanto, as questões postas apontam para a necessidade de traçarmos diretrizes para o uso responsável de aplicativos que envolvam algoritmos cada vez mais compreendidos como extensões da mente e, por conseguinte, do nosso self.

Ademais, é necessário analisar a tríade dos problemas mais comuns e discutidos quando se trata do ambiente digital e da tecnologia: a questão da privacidade, da transparência e o uso de dados sem consentimento.

\section{Privacidade, transparência e o uso de dados sem consentimento}

Muitos usuários de smartphones não têm ciência de que, enquanto acessam seus aplicativos em seus celulares, podem estar fornecendo seus dados geográficos, de provedores, preferências de acessos, operadoras de celulares sem consentimento. Avançando um pouco mais nesta questão, lembramos que nossas crenças e desejos, estados mentais complexos e bastante particulares, que estão ligados às idealizações e projeções que realizamos para compor nossas narrativas identitárias, estão sendo expostos, cotidianamente, ao visitarmos lojas virtuais. Mesmo os especialistas em tecnologia não sabem tudo sobre as combinações dos algoritmos e seus mecanismos internos de seleção, decupagem e análise, principalmente quando está envolvido o aprendizado profundo, o que leva ao problema da "transparência e da explicabilidade" (COECKELBERGH, 2019).

Para ilustrar esse tópico, temos o caso emblemático do aprendizado de máquina apresentado pelo jornal The New York Times, em 2012, com a matéria intitulada ${ }^{12}$ How Companies Learn Your Secrets, que se tornou um marco na última década do jornalismo tech. A reportagem apresenta a concepção de algoritmos baseados em aprendizado profundo que conseguem realizar predições comportamentais fundamentadas em determinados padrões de consumo e hábitos estabelecidos.

O caso, em especial, trata da loja de departamento Target que, após treinamento e constante análise de dados se tornou capaz de fornecer um modelo preditivo (predictive model) sobre determinados comportamentos. Na reportagem (DUHIGG, 2012), o jornalista menciona que ao entrevistar um funcionário da Target forneceu um exemplo hipotético para apresentar a acurácia da capacidade preditiva da empresa. 
Take a fictional Target shopper named Jenny Ward, who is 23, lives in Atlanta and in March bought cocoa-butter lotion, a purse large enough to double as a diaper bag, zinc and magnesium supplements and a bright blue rug. There's, say, an 87 percent chance that she's pregnant and that her delivery date is sometime in late August. What's more, because of the data attached to her Guest ID number, Target knows how to trigger Jenny's habits. They know that if she receives a coupon via e-mail, it will most likely cue her to buy online. They know that if she receives an ad in the mail on Friday, she frequently uses it on a weekend trip to the store. And they know that if they reward her with a printed receipt that entitles her to a free cup of Starbucks coffee, she'll use it when she comes back again (DUHIGG, 2012, versão digital).

A passagem acima mostra como, através do aprendizado e padrões estabelecidos pela equipe de estatística que programou a inteligência artificial da Target, a loja foi capaz de conceber um modelo preditivo comportamental e "deduzir" que a jovem estaria grávida e, por isso, o catálogo chegaria a sua casa, mesmo sem o acesso direto de artigos para bebês.

As Pole's computers crawled through the data, he was able to identify about 25 products that, when analyzed together, allowed him to assign each shopper a "pregnancy prediction" score. More important, he could also estimate her due date to within a small window, so Target could send coupons timed to very specific stages of her pregnancy (DUHIGG, 2012, versão digital).

O caso, apesar de emblemático, é ainda obscuro e muitas vezes discutido como apenas um case de sucesso. Todavia, o ponto que destacamos aqui é que, atualmente, esse tipo de realidade preditiva está totalmente presente em nossa ecologia informacional e é capaz de mensurar cada vez mais precisamente e, ainda, apresentar mais opções de artigos para compra. Em diversos momentos de nossas navegações somos recomendados a revisitar páginas de sites com produtos procurados anteriormente, interagir com sites que se alinhem com nosso perfil de busca, além de ofertas de cursos sobre determinado assunto que foi pesquisado anteriormente.

A internet, apesar de ser uma rede ampla e com diversas possibilidades e conteúdos disponíveis para acesso, pode ser uma armadilha para o usuário médio, que se torna uma presa fácil em um mercado que procura por nichos e fidelização de clientes. Por isso, a repetição de recomendações atua como um elemento de reforço constante que pode afetar, tanto o senso de imagem de si, quanto o acesso aos conteúdos sobre os quais o usuário procura se informar ${ }^{13}$.

Nossos dados de visitas geram ferramentas para negócios e ofertas de produtos. Nossa navegação seja em sites ou aplicativos não possui a 
transparência necessária para os usuários médios. Assim, nossa privacidade é acessada, muitas vezes sem autorização, e devolvida para nós mesmos através de ofertas que se mascaram com a rubrica de "personalização".

Desta forma, a maneira como estão configurados nossos acessos mostra um efeito em cascata: se não temos privacidade para operacionalizar nossas buscas e outras tarefas rotineiras em nossos aparelhos celulares, e não temos transparência total sobre como utilizam nossos dados, então os dados estão sendo utilizados sem nosso consentimento. A prestação de contas pelas empresas de tecnologia sobre o que é realizado com nossos dados precisa ser transparente e efetiva ${ }^{14}$.

Assumindo que nossas mentes se expandem para o mundo e se amalgamam com nossos aparelhos eletrônicos, torna-se imprescindível apresentar algumas diretrizes que auxiliem o tratamento das questões neuroéticas emergentes.

\section{Diretrizes éticas propostas}

Como mencionado anteriormente, as questões neuroéticas apresentadas para a formação do self dentro do ambiente digital estão interligadas. Contudo, realizamos um recorte sistemático para fins didáticos e de argumentação. Isto é, não há como considerar apenas uma fatia do problema, enquanto outras ações não forem implementadas. Apresentamos, assim, um conjunto de problemas, num quadro mais amplo, a fim de que o leitor possa, neste momento, concatenar todos os assuntos abordados nas seções anteriores de forma macroscópica. Destacamos quatro problemas e indicamos um caminho ético para a solução dos mesmos.

\section{Literacia digital}

Primeiramente, devemos compreender que o termo literacia não deve ser confundido apenas com a capacidade dos usuários saberem operacionalizar equipamentos tecnológicos com fluidez. Não se trata apenas da educação de pessoas que não são nativas digitais, ou seja, aquelas que nasceram antes da implementação da revolução tech e internet. Quando tratamos de literacia digital, os usuários - sejam os nativos digitais sejam os não nativos digitais - devem ter noção de todo o quadro que está por trás do uso de aplicativos: o uso de suas informações, a formação mercadológica dos nichos através dos aplicativos que são baixados em sua loja de aplicativos, bem como suas pesquisas na internet. Pouco a pouco, a personalização pelas recomendações e utilização das ferramentas digitais e TMEs "fecha a Gestalt" do próprio sujeito, sem que o usuário se dê conta de que está sendo direcionado.

Como mencionamos antes, não defendemos a abolição de aplicativos, mas que todos os usuários tenham conhecimento de que o que é entregue dentro das redes, suas informações, são as principais porções de sua identidade, e que o resultado, quando não refletido, pode ser uma completa 
entrega de seu self. Os conteúdos assimilados e personalizáveis cada vez mais devem ter o pensamento crítico adicionado à compreensão de que esses algoritmos são muletas cognitivas, mas que servem a dois propósitos: (1) nos ajudar e realizar um processo de economia cognitiva cada vez mais ágil, mas, também (2) capturar informações que servem como metrificação e construção de nichos de mercado.

\section{Problema global}

Em todas as regiões do planeta, mesmo com todas as discrepâncias do Índice de Desenvolvimento Humano (IDH), estamos cada vez mais digitais. Neste sentido, grande parte da nossa identidade pessoal recebe influência direta dos algoritmos e o problema neuroético da privacidade e do consequente uso de dados, deveria ser tratado de forma global, tal como os debates ambientais. Entretanto, a regra atual tem sido a de que cada país, com suas regras e marcos legais, acabe, ou não, se blindando, nesse quesito ${ }^{15}$.

A proteção de dados dos usuários, além da compreensão através da literacia, deve ter um respaldo jurídico eficiente e capaz de proteger aqueles usuários que não alcançaram fluência nas redes, bem como aqueles que estão em dia com sua segurança de dados. Neste sentido, é necessária a construção de protocolos e acordos entre os diversos países para o controle do uso dos dados e regras, bem demarcadas, capazes de oferecer proteção e vigilância às ações tomadas pelas empresas tecnológicas.

\section{Postura ética ativa}

Uma das diretrizes que devem ser implementadas para a construção de aplicativos e outros serviços deve ser a inclusão da reflexão da ética durante o processo de concepção do código, bem como das funções que ele realizará. É bastante comum, como foi o caso que apresentamos da Target, que as consequências éticas e legais sejam vistas apenas após o fato realizado. Isso significa que as regras, sanções e imposições legais surgem apenas depois do aplicativo consolidado. Propomos, tal como Coeckelbergh (2019), uma abordagem proativa para que exista uma regulação no projeto, uma ética da Inteligência Artificial consistente que possa não apenas sanar problemas que aconteceram, mas antecipar problemas e prevê-los, como uma medida preventiva. Em outras palavras, a abordagem ética deve estar em jogo desde os primeiros momentos da criação da estrutura tecnológica e não apenas na averiguação de benefícios e/ou malefícios.

Uma possibilidade para tal regulamentação é a submissão de projetos para Comissões de Ética, tal como os projetos de pesquisa que envolvem seres humanos. Sabemos que por se tratar de um campo extremamente novo, volátil e em franca expansão, buscar esse tipo de Comissão seria um segundo passo, após a realização de acordos globais, tal qual mencionamos no tópico anterior. Outrossim, é possível vislumbrar esse tipo de abordagem, uma vez que ao compreendermos nossa identidade pessoal 
como baseada neste caleidoscópio biológico-tecnológico, necessitamos tanto de análises mais apuradas, como de mais cuidado na criação dos projetos tecnológicos.

\section{Trabalho interdisciplinar}

Por fim, para que essas diretrizes sejam implementadas é necessário um trabalho em equipe que realize uma interface entre os profissionais da tecnologia e os das humanidades. O trabalho em conjunto deve ser uma premissa básica para esse tipo de temática. Para Coeckelbergh (2019) ainda existe uma lacuna grande entre os profissionais das humanidades e aqueles com background técnico. Ainda, segundo o autor, "essa lacuna também pode atrapalhar a elaboração de políticas em áreas como a Ética da Inteligência Artificial" (2019, 33).

\section{Considerações finais}

A efetividade da criação desses encaminhamentos apontados depende da criação de espaços para a discussão, onde seja possível tratar os problemas neuroéticos, as futuras diretrizes éticas, bem como, o próprio processo de formação educacional de manejo das tecnologias, entendendo os riscos, compromissos e atribuições que estão sendo simultaneamente gerados com os aplicativos. Neste sentido, buscamos ressaltar o quanto o domínio da pauta neuroética é crucial para a formação do self ou identidade pessoal.

Assumindo que as TMEs são parte de nossas mentes e de que devemos dar atenção às questões da autenticidade e da autonomia na própria criação da identidade pessoal, destacamos a necessidade de compreender o que pode ser realizado através das ferramentas tecnológicas e algoritmos, referente ao problema da quantificação do self. Neste contexto, ao imaginarmos uma situação em que o usuário não esteja alinhado à tecnologia, ou que surja algum problema, aportamos no campo da atribuição da responsabilidade. A quem poderíamos atribuir a responsabilidade sobre algum malefício que fosse causado ao sujeito?

Afloram, neste cenário, questões sobre (1) privacidade; transparência acerca do modo de utilização das informações do usuário e (3) sobre o uso sem consentimento dessas informações. Procuramos oferecer algumas diretrizes que possibilitem um olhar anterior ao enfrentamento de uma situação-limite, um cenário de prevenção e educação, em que possamos atuar, antes de nos depararmos com impasses éticos de maiores proporções. 


\section{Notas}

${ }^{1}$ Doutor em Filosofia pela Universidade Federal do Rio de Janeiro (UFRJ). Realiza pós-doutorado no Programa de Pós-graduação em Bioética, Ética Aplicada e Saúde Coletiva (PPGBIOS) na Universidade Federal do Rio de Janeiro (UFRJ). Atua na área de Filosofia da Mente e da Tecnologia e seus desdobramentos na Neuroética. ORCID-iD: $\quad$ https://orcid.org/0000-0002-6032-1389; e-mail: diogomochcovitch@gmail.com

2 Doutora em Filosofia pela Freie Universität Berlin (1993) e Pós-Doutora na Universidade de Connecticut (2003), na Universidade de Oxford (2006/2007), na Universidade de Tulane (2015) e na Universidade Rey Juan Carlos (2019). Professora titular da Universidade Federal do Rio de Janeiro, onde integra o Departamento de Filosofia e o Programa Interinstitucional e Interdisciplinar de PósGraduação em Bioética, Ética Aplicada e Saúde Coletiva. ORCIDiD: https://orcid.org/0000-0002-4689-9256; e-mail: mcdias1964@gmail.com

3 Para uma discussão pormenorizada da teoria da mente estendida, avaliação de seus pressupostos, assim como suas ramificações, ver: Da mente ao self estendido: a integração de artefatos na constituição da identidade pessoal (MOCHCOVITCH, 2020) e Perspectiva dos funcionamentos: fundamentos teóricos e aplicações (DIAS, 2019).

4 Segundo o relatório de 2019 da GSMA Foundation, o número de usuários de aparelhos móveis no mundo corresponde a 5,1 milhões (67\% da população mundial) e 9 milhões de dispositivos IoT (Internet of Things). Do quantitativo de aparelhos móveis, os smartphones já correspondem a $60 \%$, com projeção de $79 \%$ para 2025.

${ }^{5}$ Em Da mente ao self estendido: a integração de artefatos na constituição da identidade pessoal (MOCHCOVITCH, 2020), foi analisado detidamente como artefatos podem ser considerados partes constitutivas do self.

${ }^{6}$ As características constitutivas devem se comportar de acordo com as restrições da realidade (narrativas devem estar de acordo com as leis naturais. Seres humanos não podem voar, nem andar sobre a água, por exemplo) e das restrições de articulação (a história que identifica o sujeito deve ser articulada. Não posso ser um enxadrista campeão mundial, sem sequer ter conhecido as regras do xadrez, por exemplo).

7 Não temos a intenção de defender que a tecnologia per se é a totalidade do processo cognitivo, mas, como um processo que não se restringe ao âmbito corporal, é possível estabelecer que parte do processo está situado fora do domínio biológico.

${ }^{8}$ A visão de mente defendida aqui tem viés funcionalista. O funcionalismo defende uma visão materialista que não aceita a forma cartesiana de dualismo mente/corpo e admite a possibilidade da múltipla realização, ou seja, diversos artefatos podem executar a mesma função, dispensando a necessidade de uma base biológica específica. 
9 Vontades ou desejos são comumente divididos em primeira e segunda ordem. Grosso modo, os desejos de primeira ordem são formados por desejos diretos de obter ou realizar algo, como saciar sede, fome, etc. Já os desejos de segunda ordem são estados mentais que refletem sobre os desejos de primeira ordem, impondo-se aos mesmos. Por exemplo: se uma pessoa tem o desejo de comer um bolo de chocolate, mas estabeleceu previamente que está em uma dieta de baixa caloria, seu desejo de segunda ordem suprime sua volição imediata, pois deseja manter sua alimentação controlada. Para mais detalhes, consultar FRANKFURT, 1971.

10 Luciano Floridi (2014), inclusive, afirma que a nossa ideia de pós-humano não deveria ser o ciborgue, mas sim ao inforg, isto é, um organismo informacional, "mutuamente conectado e incorporado em um ambiente informacional" (2014, 94).

11 A questão parece levar aos debates de substituição do trabalho baseado no aprendizado de máquina para a substituição humana. De fato, esse é um debate importante, entretanto não compete a esse texto tratar sobre esse tipo de problemática.

12 Para acesso à matéria completa acessar:

https://www.nytimes.com/2012/02/19/magazine/shopping-habits.html

13 Para uma discussão interessante sobre a formação de bolhas na internet, assistir o documentário, $O$ dilema das redes, disponível na plataforma de streaming Netflix.

14 No Brasil, temos dois importantes momentos em termos jurídicos: o primeiro momento com a aprovação da lei $n^{\circ} 12.965 / 2014$, conhecida como Marco Civil da Internet, que é a lei responsável pela regulação da internet no território brasileiro, estabelece princípios, garantias, direitos e deveres para o uso de internet no Brasil; o segundo momento foi em setembro de 2020, quando entrou em vigor a lei 13.709/2018, conhecida como Lei Geral de Proteção de Dados, LGPD. Enquanto o Marco Civil da Internet propõe um ambiente mais seguro, a LGPD foca diretamente na segurança dos dados transmitidos nesse ambiente.

15 O Parlamento europeu instituiu o European General Data Protection Regulation (GDPR). A Europa é considerada o território com imposições mais severas e com a proteção de dados mais efetiva para aos seus cidadãos e usuários de smartphones no mundo. Exemplo dessa proteção foi o bloqueio recebido pelo aplicativo WhatsApp em fevereiro de 2021. Apenas em território europeu, os usuários estão protegidos na política de privacidade do aplicativo de que seus dados não serão utilizados para gerar conteúdos de vendas personalizáveis e para fins de conhecimento de mercado. Sobre esse tema, a informação está disponível na própria página do aplicativo: https://www.whatsapp.com/legal/updates/privacy-policy/?lang=pt br. 


\section{Referências bibliográficas}

ADAMS, F.; AIZAWA, K. "The bounds of cognition", in: Philosophical psychology 14.1: 43-64, 2001.

BRASIL. Lei no. 12.965, de 24 de abril de 2014. Estabelece princípios, garantias, direitos e deveres para o uso da Internet no Brasil. Disponível em: http://www.planalto.gov.br/ccivil 03/ ato2011-

2014/2014/lei/l12965.htm

BRASIL. Lei no 13.709, de 14 de agosto de 2018. Lei Geral de Proteção de Dados (LGPD). Disponível em:

http://www.planalto.gov.br/ccivil 03/ ato2015-

2018/2018/lei/L13709.htm

CARTER, J. D.; PALERMOS, O. "Is Having Your Computer Compromised a Personal Assault? The Ethics of Extended Cognition", in: Journal of the American Philosophical Association, v.2, n.4, p. 542-560, dec. 2016.

CLARK, A; CHALMERS, D. "The Extended Mind", in: Analysis 58: 10-23, 1998.

CLARK, A. Being There: Putting Brain, Body and World Together Again, Cambridge: MIT Press, 1997.

CLARK, A. Mindware: an introduction to the philosophy of cognitive Science, New York: Oxford University Press, 2001.

CLARK, A. Natural-Born Cyborgs. Minds, Technologies, and the Future of Human Intelligence, New York: Oxford University Press, 2003.

CLARK, A. "Memento's revenge: the extended mind, extended", in: MENARY, Richard. The extended mind (Life and Mind: Philosophical Issues in Biology and Psychology), Cambridge: MIT Press, 2010.

COECKELBERGH, M. "Ethics of artificial intelligence: Some ethical issues and regulatory challenges", in: Technology and Regulation, v. 2019, n. 01, p. 31-34, 2019. Disponível em: https://doi.org/10.26116/techreg.2019.003

DIAS, M. C. Perspectiva dos funcionamentos: fundamentos teóricos e aplicações. Rio de Janeiro: Ape'Ku, 2019.

DENNETT, D. Tipos de mentes: rumo a uma compreensão da consciência. Tradução: Alexandre Tort, Rio de Janeiro: Rocco, 1997. 
DUHIGG, C. "How Companies Learn Your Secrets". The New York Times, Nova Iorque, 16 de fevereiro de 2012. Disponível em: https://www.nytimes.com/2012/02/19/magazine/shopping-habits.html

ERDENIZ, S.; MENYCHTAS, A.; MAGLOGIANNIS, I; FELFERNIG, A.; TRAN, T. "Recommender systems for IoT enabled quantifed-self applications", in: Evolving Systems, v. 10, n. 40, p. 1-14, 2019.

EUROPA. Regulamento (UE) 2016/679 do Parlamento Europeu e do Conselho de 27 de abril de 2016. Relativo à proteção das pessoas singulares no que diz respeito ao tratamento de dados pessoais e à livre circulação desses dados e que revoga a Diretiva 95/46/CE (Regulamento Geral sobre a Proteção de Dados). Disponível em: https://eur-lex.europa.eu/legalcontent/PT/TXT/HTML/?uri=CELEX:32016R0679\&from=PT

FLORIDI, L. The fourth Revolution: how infosphere is reshaping human reality. Oxford: Oxford University Press, 2014.

FRANKFURT, H. "Freedom of the will and the concept of a person" in.: The Journal of Philosophy, v. 6, n. 1, p. 5-20, jan. 1971.

GABRIELS, K.; COECKELBERGH, M. "Technologies of the self and other: how self-tracking technologies also shape the other", in: Journal of Information, Communication and Ethics in Society, v.17, n.2, p. 119 - 127, 2019. Disponível em: https://doi.org/10.1108/JICES-12-2018-0094

GIBSON, J. The ecological approach to visual perception. New York: Taylor and Francis Group, 1986.

GSMA. The mobile economy 2019, 2019. Disponível em: https://www.gsma.com/r/mobileeconomy/.

HUSSERL, E. A ideia da fenomenologia. Tradução: Artur Morão. Lisboa: Ed. 70, 2000.

KREITMAIR, K.; CHO, M. C. "The neuroethical future of wearable and mobile health technology", in: Illes, J. Neuroethics: antecipating the future, Oxford: Oxford University Press, 2017.

LÉVY, P. As tecnologias da Inteligência: o futuro do pensamento na era da informática. São Paulo. Editora 34. Tradução de Carlos Irineu da Costa. 1993.

MERLEAU-PONTY, M. Fenomenologia da percepção. Tradução: Carlos Alberto Ribeiro de Moura. São Paulo: Martins Fontes, 2006. 
MOCHCOVITCH, D. Da mente ao self estendido: a integração de artefatos na constituição da identidade pessoal. Rio de Janeiro: Ape'Ku, 2020.

REINER, P. B.; NAGEL, S. K. "Technologies of the extended mind: defining the issues", in: Illes, J. Neuroethics: antecipating the future, Oxford: Oxford University Press, 2017.

RUPERT, R. Cognitive systems and the extended mind. New York: Oxford University Press, 2009.

SCHECHTMAN, MARYA. The constitution of selves. Nova Iorque: Cornell University Press, 1996.

THE SOCIAL DILEMMA. Jeff Orlowski. Califórnia: Netflix, 2020. Documentário disponível em plataforma digital.

WILSON, R. Boundaries of the mind: the individual in the fragile sciences. New York: Cambridge University Press, 2004. 